\title{
Covering the gastric tube with the mediastinal pleura during minimally invasive McKeown esophagectomy can reduce the incidence of anastomotic fistulae
}

\author{
Xiaodong Zhu ${ }^{1,2}$, Hanran $\mathrm{Wu}^{2}$, Changqing $\mathrm{Liu}^{2}$, Xinyu $\mathrm{Mei}^{2}$ \\ ${ }^{1}$ Department of Thoracic Surgery, Anhui Provincial Hospital, Wannan Medical College, Hefei, China \\ 2Department of Thoracic Surgery, The First Affiliated Hospital of USTC, Anhui Provincial Hospital, Division of Life Sciences \\ and Medicine, University of Science and Technology of China, Hefei, China
}

Videosurgery Miniinv 2021; 16 (3): 612-619

DOI: https://doi.org/10.5114/wiitm.2021.105155

\begin{abstract}
Introduction: The rate of anastomotic leakage from intrathoracic esophagogastric anastomoses can be reduced by covering them with the mediastinal pleura. Whether anastomotic leakage can be reduced by covering the portion of the gastric tube in the upper mediastinum with the mediastinal pleura during minimally invasive McKeown esophagectomy (MIE McKeown) is unknown.

Aim: To evaluate the consequence of covering the mediastinal pleural during minimally invasive McKeown esophagectomy.

Material and methods: Consecutive patients who underwent MIE McKeown between January 2015 and December 2019 were retrospectively analyzed. Participants for whom the portion of the gastric tube in the upper mediastinum was not covered with the mediastinal pleura were assigned to group $A$; otherwise, they were assigned to group $B$. Chi-square analysis and univariable and multivariable logistic analyses were used to compare the differences between the two groups and explore the risk factors for anastomotic fistulae.

Results: A total of 267 patients with middle and lower esophageal cancer were included in this study (131 in group $A$ and 136 in group B). Anastomotic leakage occurred in 5 patients (5/136) in group B compared with 13 patients $(13 / 131)$ in group $A(p=0.042)$. Univariable and multivariable logistic analyses identified a gastric tube not covered with the mediastinal pleura as a risk factor for significantly greater anastomotic leakage $(p=0.042)$, but it was not an independent prognostic factor for anastomotic leakage (odds ratio $=0.585,95 \%$ confidence interval: lower bound: 0.069, upper bound, 1.122).

Conclusions: This study provides preliminary evidence that covering the gastric tube with the mediastinal pleura during MIE McKeown can decrease the incidence of anastomotic leakage.
\end{abstract}

Key words: covering mediastinal pleura, McKeown esophagectomy, anastomotic leakage.

\section{Introduction}

Esophageal cancer is the sixth leading cause of cancer-related mortality worldwide and is ranked fourth in China [1, 2]. Surgical treatment is the primary choice for nonmetastatic esophageal cancer ac- cording to international guidelines [3, 4]. Minimally invasive surgical approaches, which result in smaller sutures, fewer postoperative complications and faster recovery than more open surgical approaches, have been widely accepted by both patients and sur-

\section{Address for correspondence}

Prof. Xinyu Mei, Department of Thoracic Surgery, The First Affiliated Hospital of USTC, Anhui Provincial Hospital, Division of Life Sciences and Medicine, University of Science and Technology of China, Hefei, China, phone: +86 5516228 3326, e-mail: mxyahslyy@163.com 
geons. Minimally invasive McKeown esophagectomy (MIE McKeown), i.e., thoraco-laparoscope-assisted esophagectomy with left cervical anastomosis, is a mainstream surgical technique for esophageal malignancies that can achieve a wider oncological resection margin and better lymph node harvest than intrathoracic anastomoses or transhiatal esophagectomy $[4,5]$. However, the main disadvantage of MIE Mckeown is anastomotic fistulae, which can prolong hospitalization and increase the occurrence of anastomoses and anastomotic stenosis [6].

Although many modified measures have been used during MIE McKeown, such as suturing the anastomosis or covering the anastomosis with pedicled omentum, few satisfactory results have been obtained [7]. Asteriou et al. analyzed 57 patients whose esophagogastric anastomoses were covered with the mediastinal pleura during Ivor-Lewis esophagectomy, which significantly reduced the incidence of anastomotic leakage [8]. However, few studies have examined whether a modified mediastinal pleura can reduce anastomosis leakage during MIE McKeown.

\section{Aim}

The goal of this study was to evaluate the feasibility and safety of covering the gastric tube with the mediastinal pleura in MIE McKeown and evaluate whether it can reduce the incidence of anastomotic leakage.

\section{Material and methods}

\section{Study design and clinical data}

This study was approved by the medical ethics committee of the hospital, which waived the requirement of informed consent of the patients. The study reviewed patients who underwent MIE McKeown with esophageal cancer between January 2015 and December 2019 based on the following criteria: (I) a diagnosis of middle or lower esophageal cancer according to pathology results; (II) treatment with MIE McKeown operation with mechanical stapler anastomosis and two-field lymphadenectomy (posterior mediastinum, upper abdomen); and (III) a clinical stage of T1-3N0-1M0 according to the 8th edition of the American Joint Committee on Cancer (AJCC) Tumor Node Metastasis (TNM) staging system. The exclusion criteria were as follows: (I) diagnosis of upper esophageal cancer treated with tumor en bloc resection and three-field lymphadenectomy; (II) history of esophageal or stomach surgery; (III) treatment with neoadjuvant therapy; and (IV) a lack of perioperative clinical data. According to these criteria, $267 \mathrm{pa}$ tients were ultimately reviewed (Figure 1), including 131 patients who underwent MIE McKeown without covering the mediastinal pleural on the gastric tube between January 2015 and December 2016 (group A) and 136 patients who underwent MIE McKeown with covering the mediastinal pleural on the gastric tube between January 2017 and December 2019 (group B). All participants received standard preoperative evaluations, such as upper gastrointestinal radiography, abdominal-thoracic computed tomography $(\mathrm{CT})$, esophagography and echocardiography.

\section{Surgical procedure}

Participants underwent MIE McKeown with twofield lymphadenectomy. Each patient was placed in the left lateral decubitus position after double-lumen endotracheal intubation anesthesia. The observation port was located in the $7^{\text {th }}$ intercostal space on the posterior axillary line, the main operating port was located in the $4^{\text {th }}$ intercostal space on the anterior axillary line, and the secondary operating port was placed in the $8^{\text {th }}$ intercostal space on the subscapular line.

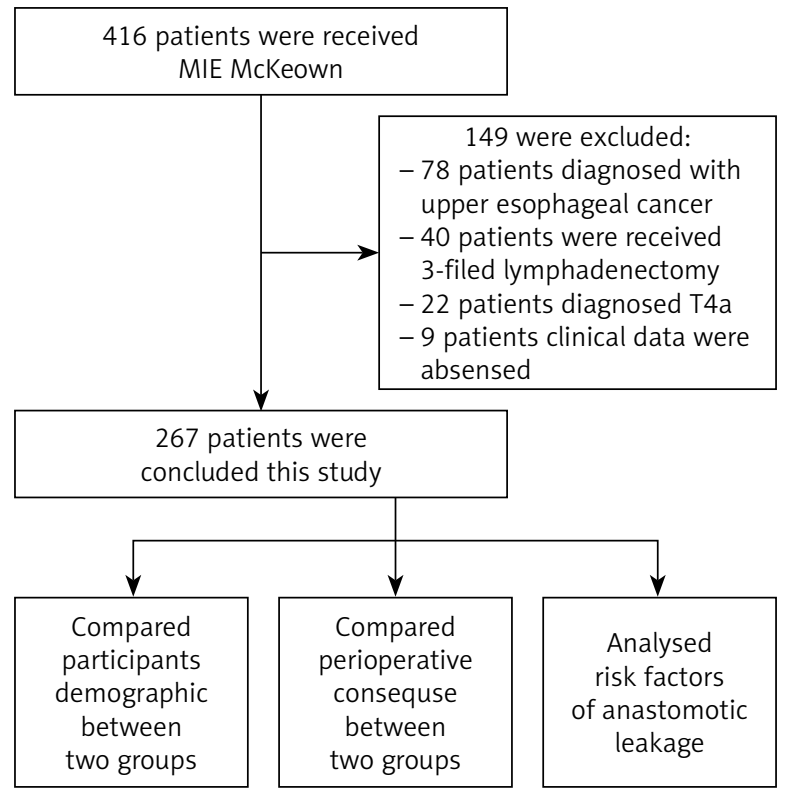

Figure 1. Flowchart of the study. MIE: minimally invasive esophagectomy 
The right recurrent laryngeal nerve (RLN) and vagus nerve were exposed first, followed by division of the mediastinal pleura. The azygos vein was occluded by a Hem-o-lok clip, and the entire thoracic esophagus was divided with an ultrasound scalpel. We used blunt dissection to remove the lymph nodes adjacent to the left and right RLNs. Other lymph nodes were dissected according to the criteria of the $8^{\text {th }}$ edition of the AJCC TNM staging system [9]. We did not typically remove the esophagus after the completion of the thoracic operation. Occasionally, for cases in which the volume of the tumor was too large to pass through the thoracic inlet, we would use a specimen retrieval bag to remove the tumor from the thoracic cavity.

Group A: after dissociation of the esophagus, a chest tube was finally placed for drainage through the operation port, completing the thoracic procedure (Photo $1 \mathrm{~A}$ ).

Group B: after dissociation of the thoracic esophagus and regional lymphadenectomy, the mediastinal pleura was sutured from the azygos vein to the top of the thoracic cavity every centimeter. Finally, a chest tube was placed to complete the thoracic procedure (Photo $1 \mathrm{~B}$ ).

The laparoscopy was performed as described previously [10]. The final step was cervical anastomosis. An incision was made in the blunt free muscle tissue at the medial edge of the left sternocleidomastoid muscle along with simultaneous exploration of the cervical lymph node region. The gastric tube was pulled up to the neck, and an end-to-side anastomosis was performed using a circular stapler (Ethicon, Somerville, NJ, USA). The operation was completed after insertion of a nasointestinal tube and a nasogastric tube.

\section{Postoperative management and definition of postoperative complications}

All patients received total parenteral nutrition on the first postoperative day and partial enteral nutrition from the nasoduodenal tube on the second postoperative day. We evaluated the healing of the gastroesophageal anastomoses by sensitive and noninvasive CT examination on the seventh postoperative day. Anastomotic fistulae were mainly identified according to their clinical and radiological signs. Clinical signs included sepsis and cervical sutures showing signs of infection or leakage of clear fluid, while radiological signs included mediastinal cysts, pneumomediastinum, and mediastinal abscesses [11]. Other postoperative complications were evaluated in accordance with the Esophageal Complications Consensus Group (ECCG) system [11, 12]. Complications were stratified according to the Clavien-Dindo classification system into minor (Clavien-Dindo grades $\mathrm{I}-\mathrm{II}$ ) and major (Clavien-Dindo grades III-V) events [13].

If there were no clinical manifestations of anastomotic fistulae, the patient was gradually transitioned from a liquid diet to a normal diet. Then, the chest tube was removed when the thoracic drainage was less than $100 \mathrm{ml} /$ day and postoperative X-ray showed the absence of pleural effusion. If there were clinical manifestations of anastomotic fistula, the patient presented with fever, or drainage color changes were observed during the liquid diet period, a radiological water-soluble contrast study was performed.

The patient was discharged on postoperative day 10-11 after the drainage tube was removed if there were no postoperative complications.
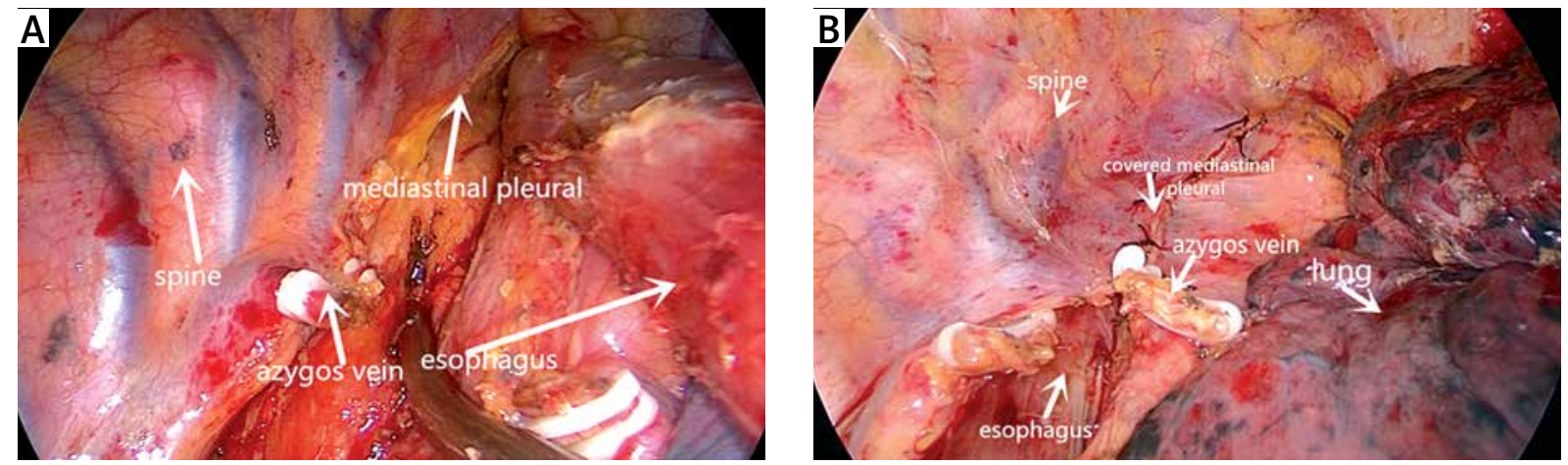

Photo 1. A - The mediastinal pleura was not sutured after the thoracic procedure. B - Suturing the mediastinal pleural after the thoracic procedure 


\section{Statistical analysis}

Categorical variables are presented as $n(\%)$, and differences were compared by the Mann-Whitney $U$ test, $\chi^{2}$ test or Fisher's exact test. A two-tailed, unpaired $t$-test was used to compare continuous variables. Binary logistic regression models were used to explore the risk factors for anastomotic fistulae. A two-sided $p$-value of less than 0.05 was considered statistically significant. Statistical analyses were performed using SPSS20.0 software.

\section{Results}

\section{Patient characteristics}

The procedures performed on the patients throughout the trial are described in Figure 1. The average patient age was $66.71 \pm 8.28$ years old; 204 (76.4\%) patients were male, and $63(23.6 \%)$ were female. We sorted 131 (49.1\%) patients whose gastric tubes were not covered with the mediastinal pleura into group A, and the remaining 136 (50.9\%) patients, whose gastric tubes were covered with mediastinal pleura, were included in group B.

The clinical characteristics of the two groups are displayed in Table I. No patient was converted from MIE McKeown to open surgery. Group B had a longer operative time (302.01 \pm 47.59 vs. $284.92 \pm 58.01$ min, $p=0.01)$ but a shorter duration of thoracic drainage (6.79 \pm 3.00 vs. $7.50 \pm 2.46$ days, $p=0.038)$ and hospital stay $(13.15 \pm 4.74$ vs. $14.76 \pm 4.75$ days, $p<0.01)$ than group A. There were no significant differences in terms of blood loss, duration of nasogastric tube placement or lymph nodes harvested between the two groups. The details for these parameters are summarized in Table II.

\section{Postoperative complications}

The total incidence of postoperative complications was $27.0 \%$ (group A, 29.8\%; group B, $24.3 \%$ ); the details are outlined in Table III. A patient in group B had undergone pacemaker surgery 18 years previously and died of heart failure 18 days after the operation.

The incidence of anastomotic leakage in group $B$ was significantly lower than that in group A: 5 of 136 (3.7\%) versus 13 of 131 (9.9\%), retrospectively ( $p=$ $0.042)$. Thirty-one $(23.7 \%)$ patients in group $A$ and 19 patients in group B (14.0\%) suffered from postoperative pneumonia; this difference was significant.
There was no significant difference between the two groups in terms of the incidence of major complications (group A, $11.6 \%$; group $\mathrm{B}, 6.6 \% ; p=0.168$ ) or minor complications (group A, $18.3 \%$; group $B$, $17.6 \% ; p=0.886)$.

\section{Risk factors for anastomotic fistulae}

To identify the risk factors and independent risk factors for anastomotic fistulae, we used a binary logistic regression model. In the univariable analysis, current smoking (odds ratio $(O R)=4.07,95 \%$ confidence interval (Cl): 1.41-11.79; $p<0.01)$ and a gastric tube that was not covered with mediastinal pleura $(\mathrm{OR}=0.346,95 \% \mathrm{Cl}: 0.120-1.001 ; p=$ 0.042 ) were risk factors for anastomotic fistulae. In the multivariable analysis, a gastric tube not covered with mediastinal pleura ( $\mathrm{OR}=0.59 ; 95 \% \mathrm{Cl}: 0.069-$ 1.12; $p=0.72$ ) was not an independent risk factor for anastomotic leakage (Table IV).

\section{Discussion}

MIE McKeown was first performed in the 1990s, and many thoracic surgeons currently initially choose this method to treat esophageal cancer [14]. Compared with other MIE procedures, MIE McKeown is associated with a greater number of postoperative complications, such as anastomotic leakage and postoperative pneumonia, which are directly related to postoperative morbidity and even mortality $[15,16]$.

Anastomotic leakage, a serious postoperative complication of MIE McKeown, is associated with anastomotic tension and insufficient blood supply [17]. The occurrence of anastomotic fistulae increases perioperative morbidity and tends to lower patient quality of life after the operation. Many surgeons prefer to reinforce the anastomosis with pedicled omentum or by suturing the anastomosis after stapling $[18,19]$. Zhou et al. reinforced MIE McKeown anastomoses with pedicled omentum, improving their blood supply and reducing the incidence of anastomotic leakage to $4.6 \%$ (4/87 after reinforcement vs. $11 / 73$ before, $p=0.023$ ) [20]. However, there is a potential risk for the pedicled omentum to undergo avascular necrosis from having to share the right gastroepiploic artery blood supply [19].

Many surgeons also use the mediastinal pleura in pulmonary lobectomy to prevent air leakage and have achieved satisfactory results [21]. Consequently, we performed partial mediastinal pleu- 
Table I. Clinical and demographic characteristics of the patients

\begin{tabular}{|c|c|c|c|c|}
\hline Patient characteristics & Group A $(n=131)$ & Group B $(n=136)$ & Statistical value & $P$-value \\
\hline Age [years] & $66.73 \pm 8.61$ & $66.68 \pm 7.97$ & 0.048 & 0.962 \\
\hline Gender, $n(\%)$ : & & & 0.099 & 0.753 \\
\hline Male & 99 (75.6) & $105(77.2)$ & & \\
\hline Female & $32(24.4)$ & $31(22.8)$ & & \\
\hline Tumor location, $n(\%)$ : & & & 0.970 & 0.325 \\
\hline Middle & $94(71.2)$ & $90(66.2)$ & & \\
\hline Lower & $37(28.8)$ & $46(33.8)$ & & \\
\hline BMI $\left[\mathrm{kg} / \mathrm{m}^{2}\right]$ & $22.64 \pm 3.41$ & $22.66 \pm 2.98$ & -0.041 & 0.968 \\
\hline NRS 2002 score, $n$ (\%): & & & 0.989 & 0.320 \\
\hline$<3$ & $81(61.8)$ & $92(67.6)$ & & \\
\hline$\geq 3$ & $50(38.2)$ & $44(32.4)$ & & \\
\hline Current smoker, $n$ (\%): & & & 0.568 & 0.451 \\
\hline Yes & $57(43.5)$ & $53(40.5)$ & & \\
\hline No & $74(56.5)$ & $83(59.5)$ & & \\
\hline Pathologic staging (TNM), $n$ (\%): & & & -0.563 & 0.574 \\
\hline I & $16(12.2)$ & 27 (19.9) & & \\
\hline ॥ & $62(47.3)$ & $54(39.7)$ & & \\
\hline III & $50(38.9)$ & $50(36.8)$ & & \\
\hline IV & $2(1.5)$ & $5(3.7)$ & & \\
\hline Differentiation, $n$ (\%): & & & -1.369 & 0.171 \\
\hline High & $13(10.4)$ & $22(16.9)$ & & \\
\hline Moderate & $96(76.8)$ & $71(54.6)$ & & \\
\hline Poor & $16(12.8)$ & $37(28.5)$ & & \\
\hline Tumor histology: & & & -0.311 & 0.756 \\
\hline Squamous cell carcinoma & $121(92.4 \%)$ & $127(93.4 \%)$ & & \\
\hline Adenosquamous carcinoma & $3(3.1 \%)$ & $3(2.2 \%)$ & & \\
\hline Others* & $6(4.6 \%)$ & $6(4.4 \%)$ & & \\
\hline \multicolumn{5}{|l|}{ Pre-comorbidities, $n(\%)$ : } \\
\hline Hypertension & $23(17.6)$ & $33(24.3)$ & 1.811 & 0.178 \\
\hline Pulmonary disease & $38(29.0)$ & $34(25.0)$ & 0.544 & 0.461 \\
\hline Heart disease & $17(13.0)$ & 25 (18.4) & 1.471 & 0.225 \\
\hline Diabetes & $9(6.9)$ & $10(7.4)$ & 0.24 & 0.878 \\
\hline
\end{tabular}

Group A - not covering the mediastinal pleural on the gastric tube. Group B - covering the mediastinal pleural on the gastric tube. BMI - body mass index is the weight in kilograms divided by the square of the height in meters. NRS2002 score - Nutritional risk screening 2002 score, NRS2002 less than 3 was defined as nutritional risk. TNM - Tumor-node-metastasis, according to the diagnosis of the 8th edition of the American Joint Committee on Cancer (AJCC). Others* include adenocarcinoma, esophageal neuroendocrine carcinoma and esophagus undifferentiated carcinoma. Pulmonary disease include chronic obstructive pulmonary disease, bronchiectasis. chronic bronchitis and bronchial asthma. Heart disease include sinus arrhythmia, sinus bradycardia, coronary artery disease and chronic heart failure.

ral tenting of the gastric tube near the esophagogastric anastomosis in the current study, and the incidence of anastomotic leakage was reduced to $3.8 \%$. The incidence of anastomosis leakage was lower than that in our previous study [10]. The po- tential reasons are as follows: (i) By partially covering the gastric tube, the mediastinal pleural tent can reduce the displacement of the esophagogastric anastomosis due to gravity, possibly creating a tension-free anastomosis. (ii) A mediastinal pleu- 
Table II. Preoperative and short-term outcomes of the study

\begin{tabular}{|lccc|}
\hline Parameter & Group A $(n=131)$ & Group B $(n=136)$ & Statistical value \\
\hline Operation time [min] & $284.92 \pm 47.59$ & $302.01 \pm 58.01$ & -2.603 \\
\hline Blood loss [ml] & $134.35 \pm 83.00$ & $149.51 \pm 192.39$ & -0.831 \\
\hline $\begin{array}{l}\text { Postoperative hospital } \\
\text { stay [days] }\end{array}$ & $14.76 \pm 4.75$ & $13.15 \pm 4.74$ & 2.757 \\
\hline $\begin{array}{l}\text { Duration of chest tube } \\
\text { drainage [days] }\end{array}$ & $7.50 \pm 2.46$ & $6.79 \pm 3.00$ & 2.086 \\
\hline $\begin{array}{l}\text { Duration of stomach } \\
\text { tube drainage [days] }\end{array}$ & $9.85 \pm 3.74$ & $9.95 \pm 3.94$ & -0.215 \\
\hline $\begin{array}{l}\text { Lymph nodes har- } \\
\text { vested }\end{array}$ & $20.87 \pm 5.32$ & $21.43 \pm 7.10$ & -0.726 \\
\hline
\end{tabular}

Group A - not covering the mediastinal pleural on the gastric tube. Group B - covering the mediastinal pleural on the gastric tube.

Table III. Post-operative complications

\begin{tabular}{|c|c|c|c|c|}
\hline General statistics & Group $\mathrm{A}(n=131)$ & Group B $(n=136)$ & Statistical value & $P$-value \\
\hline $\begin{array}{l}\text { Total perioperative complica- } \\
\text { tions, } n / \text { total } n(\%)\end{array}$ & 39/131 (29.8) & $33 / 136(24.3)$ & 1.027 & 0.311 \\
\hline \multicolumn{5}{|l|}{ Surgical complication: } \\
\hline Anastomotic leakage & 13/131 (9.9) & $5 / 136(3.7)$ & 4.142 & 0.042 \\
\hline Delayed gastric emptying & $12 / 131(9.2)$ & $7 / 136(5.1)$ & 1.626 & 0.202 \\
\hline Chylothorax & $3 / 131(2.3)$ & 2/136 (1.5) & - & $0.679^{*}$ \\
\hline Wound infection & 2/131 (1.5) & $3 / 136(2.2)$ & - & $1.00^{*}$ \\
\hline Pulmonary complication & $35 / 131(26.7)$ & 29/136 (21.3) & 1.065 & 0.302 \\
\hline Pneumonia & $31 / 131(23.7)$ & 19/136 (14.0) & 4.120 & 0.042 \\
\hline Pleural effusion & $5 / 131(3.8)$ & $6 / 136(4.4)$ & 0.060 & 0.807 \\
\hline Arrythmia & 16/131 (12.2) & $12 / 136(8.8)$ & 0.817 & 0.366 \\
\hline Mortality (within 90 days) & 0 & $1(0.7 \%)$ & - & 1.000 \\
\hline \multicolumn{5}{|c|}{ Severity of complication (Clavien-Dindo classification): } \\
\hline Grade I/II & 24/131 (18.3) & 24/136 (17.6) & 0.021 & 0.886 \\
\hline Grade IIIa/IIIb & $12 / 131(9.2)$ & $6 / 136(4.4)$ & 2.393 & 0.122 \\
\hline Grade IVa/IVb, V & $3 / 131(2.2)$ & $3 / 136(2.3)$ & - & 1.000 \\
\hline
\end{tabular}

Group A - not covering the mediastinal pleural on the gastric tube. Group B - covering the mediastinal pleural on the gastric tube. ${ }^{\star}$ Fisher's exact test.

ra-covered gastric tube can create a local closed space and absorb the transudate from microanastomotic fistulae, preventing uncomfortable clinical manifestations during hospitalization. (iii) A sufficient blood supply is a basic requirement for anastomotic healing, and the microvasculature of the mediastinal pleura can improve local circulation and benefit anastomotic healing. (iv) In the present study, by covering the gastric tube, the mediastinal pleura could prevent the tube from overexpanding during breathing, which could negatively affect the esophagogastric anastomosis.
Postoperative pneumonia is another common complication of esophagectomy. The relationship between anastomotic leakage and postoperative pneumonia is controversial, however. One study reported that inadequate postoperative tissue oxygenation can influence the healing of the anastomosis, and severe postoperative pneumonia often reduces arterial blood oxygen content [22]. Some studies have also shown that some patients with cervical anastomotic fistulae suffer from pneumonia because of anastomotic transudate flowing into the thorax or inflammatory medium [23-25]. In the present study, covering 
Table IV. Univariate and multivariate analyses of risk factors for anastomotic leakage

\begin{tabular}{|c|c|c|c|c|}
\hline \multirow[t]{2}{*}{ Clinical parameters } & \multicolumn{2}{|c|}{ Univariate analysis } & \multicolumn{2}{|c|}{ Multivariate logistic regression analysis } \\
\hline & OR $(95 \% \mathrm{Cl})$ & $P$-value & Adjusted OR (95\% Cl) & $P$-value \\
\hline Age & $3.859(65.71-67.71)$ & 0.978 & $0.903(0.832-0.979)$ & 0.014 \\
\hline Gender & $0.456(0.169-1.231)$ & 0.147 & $0.044(0.006-0.326)$ & 0.002 \\
\hline Tumor location & $0.539(0.205-1.419)$ & 0.205 & - & - \\
\hline BMI & $3.174(22.26-23.05)$ & 0.108 & $0.881(0.741-1.047)$ & 0.150 \\
\hline NRS2002 score & $0.915(0.332-2.521)$ & 0.863 & $0.341(0.079-1.478)$ & 0.150 \\
\hline Current smoker & $4.074(1.408-11.788)$ & 0.006 & $67.834(7.373-624.046)$ & 0.000 \\
\hline pT stage & - & - & $1.114(0.478-2.600)$ & 0.802 \\
\hline pN stage & - & - & $1.228(0.527-2.863)$ & 0.634 \\
\hline pG stage & - & - & $0.831(0.250-2.766)$ & 0.763 \\
\hline Neoadjuvant & $1.406(0.170-11.639)$ & 0.543 & $5.824(0.340-99.810)$ & 0.224 \\
\hline Diabetes & $0.586(0.124-2.763)$ & 0.373 & $1.124(0.134-9.450)$ & 0.914 \\
\hline cardiopulmonary & $0.630(0.197-2.018)$ & 0.499 & $0.272(0.054-1.358)$ & 0.113 \\
\hline Cover mediastinal pleural & $0.346(0.120-1.001)$ & 0.042 & $0.585(0.069-1.122)$ & 0.072 \\
\hline Hypertension & $0.503(0.180-1.405)$ & 0.227 & $0.585(0.132-2.582)$ & 0.479 \\
\hline Duration & - & - & $0.993(0.980-1.006)$ & 0.296 \\
\hline Blood & - & - & $1.001(0.996-1.005)$ & 0.754 \\
\hline
\end{tabular}

OR $(95 \% \mathrm{Cl})$ - odds ratio (95\%confidence interval).

the anastomosis with mediastinal pleura prevented esophagogastric anastomotic transudate from flowing into the thorax when anastomotic leakage occurred. Additionally, covering the gastric tube with mediastinal pleura creates a local closed space, resulting in the localization and, ultimately, the absorption of microanastomosis transudate. Thus, covering the gastric tube with mediastinal pleura would lessen the effect of any microanastomotic leakage on the patient.

The limitations of this study should be mentioned. First, it was a single-center retrospective study. Moreover, there was a lack of long-term outcome assessment, and we did not evaluate whether partial subpleural blanketing of the gastric tube improved patient quality of life. Thus, a subsequent, multicenter, randomized, controlled trial is needed to confirm these conclusions.

\section{Conclusions}

In the present study, covering the gastric tube with the mediastinal pleura was found to be an effective method to prevent esophagogastric anastomotic leakage and decrease the incidence of postoperative pneumonia. The modified pleural tent is easy to perform, and the procedure is safe for the patient.

\section{Acknowledgments}

Xiaodong Zhu and Hanran Wu are co-authors with equal contribution.

\section{Conflict of interest}

The authors declare no conflict of interest.

\section{References}

1. Kamangar F, Nasrollahzadeh D, Safiri S, et al. The global, regional, and national burden of oesophageal cancer and its attributable risk factors in 195 countries and territories, 1990-2017: a systematic analysis for the Global Burden of Disease Study 2017. Lancet Gastroenterol Hepatol 2020; 5: 582-97.

2. Qiu M, Lin J, Li X, et al. Current state of esophageal cancer surgery in China: a national database analysis. BMC Cancer 2019; 19: 1064.

3. Hagens ERC, Anderegg MCJ, van Berge Henegouwen MI, et al. International survey on the management of anastomotic leakage after esophageal resection. Ann Thorac Surg 2018; 106: 1702-8.

4. Shichinohe T, Ebihara Y, Murakami S, et al. Intraluminal continuous decompression and drainage using a vacuum pump for controlling cervical anastomotic leakage after a three-field esophagectomy with a gastric pull-up. Esophagus 2016; 13: 229-33.

5. Lv F, Zhang F, Wang Z, et al. Minimally invasive McKeown esophagectomy with two-field lymph node dissection and 
manual cervical esophagogastric anastomosis. J Thorac Dis 2019; 11: 3175-9.

6. Gooszen JAH, Goense L, Gisbertz SS, et al. Intrathoracic versus cervical anastomosis and predictors of anastomotic leakage after oesophagectomy for cancer. Br J Surg 2018; 105: 552-60.

7. Wang $\mathrm{Q}$, Ping W, Cai Y, et al. Modified McKeown procedure with uniportal thoracoscope for upper or middle esophageal cancer: initial experience and preliminary results. J Thorac Dis 2019; 11: 4501-6.

8. Asteriou C, Barbetakis N, Lalountas M, et al. Modified pleural tenting for prevention of anastomotic leak after Ivor Lewis esophagogastrectomy. Ann Surg Oncol 2011; 18: 3737-42.

9. Rice TW, Ishwaran H, Ferguson MK, et al. Cancer of the esophagus and esophagogastric junction: an eighth edition staging prime. J Thorac Oncol 2017; 12: 36-42.

10. Mei X, Xu M, Guo M, et al. Minimally invasive Ivor-Lewis oesophagectomy is a feasible and safe approach for patients with oesophageal cancer. Anz J Surg 2016; 86: 274-9.

11. Goense L, Stassen PMC, Wessels FJ, et al. Diagnostic performance of a CT-based scoring system for diagnosis of anastomotic leakage after esophagectomy: comparison with subjective CT assessment. Eur Radiol 2017; 27: 4426-34.

12. Low DE, Alderson D, Cecconello I, et al. International consensus on standardization of data collection for complications associated with esophagectomy. Ann Surg 2015; 262: 286-94.

13. Dindo D, Demartines N, Clavien P. Classification of surgical complications. Ann Surg 2004; 240: 205-13.

14. Hagens ERC, Anderegg MCJ, van Berge Henegouwen MI, et al. International survey on the management of anastomotic leakage after esophageal resection. Ann Thorac Surg 2018; 106: 1702-8.

15. van Workum F, Slaman AE, van Berge HM, et al. Propensity score-matched analysis comparing minimally invasive ivor lewis versus minimally invasive McKeown esophagectomy. Ann Surg 2020; 271: 128-33.

16. Deng J, Su Q, Ren Z, et al. Comparison of short-term outcomes between minimally invasive McKeown and Ivor Lewis esophagectomy for esophageal or junctional cancer: a systematic review and meta-analysis. Onco Targets Ther 2018; 11: 6057-69.

17. Schizas D, Kosmopoulos M, Giannopoulos S, et al. Meta-analysis of risk factors and complications associated with atrial fibrillation after oesophagectomy. Br J Surg 2019; 106: 534-47.

18. Zhang S, Huang W, Liu X, et al. Pilot study on preventing anastomotic leakage in stapled gastroesophageal anastomosis. Thorac Cancer 2018; 9: 142-5.

19. Wiggins T, Markar SR, Arya S, et al. Anastomotic reinforcement with omentoplasty following gastrointestinal anastomosis: a systematic review and meta-analysis. Surg Oncol 2015; 24: 181-6.

20. Zhou D, Liu Q, Deng X, et al. Anastomotic reinforcement with omentoplasty reduces anastomotic leakage for minimally invasive esophagectomy with cervical anastomosis. Cancer Manag Res 2018; 10: 257-63.

21. McConnell PI. Extracellular matrix pleural tent for persistent air leak and air space in a child after upper lobectomy. Ann Thorac Surg 2015; 99: 321-3.
22. Goense L, van Rossum PSN, Tromp M, et al. Intraoperative and postoperative risk factors for anastomotic leakage and pneumonia after esophagectomy for cancer. Dis Esophagus 2017; 30: 1-10.

23. van Rossum PSN, Haverkamp L, Carvello M, et al. Management and outcome of cervical versus intrathoracic manifestation of cervical anastomotic leakage after transthoracic esophagectomy for cancer. Dis Esophagus 2016; 30: 1-8.

24. Cafarotti S, Cesario A, Porziella V, et al. Intrathoracic manifestations of cervical anastomotic leaks after transhiatal and transthoracic oesophagectomy . Br J Surg 2010; 97: 1745-6.

25. Korst RJ, Port J, Lee PC, et al. Intrathoracic manifestations of cervical anastomotic leaks after transthoracic esophagectomy for carcinoma. Ann Thorac Surg 2005; 80: 1185-90.

Received: 20.10.2020, accepted: 22.12.2020. 